\title{
The ethicolegal framework relevant to human faecal microbiota transplants in South Africa: Part 1. A legal vacuum
}

M Labuschaigne, ${ }^{1}$ BA Hons, MA, DLitt, LLB, LLD; M Slabbert, ${ }^{1}$ BA Hons, HED, BProc, LLB, LLD; S Budree, ${ }^{2}$ MB ChB, DCH, FC Paed (SA), Cert Gastroenterology (SA) Paed; E Hoosien, ${ }^{3}$ MB ChB, MMed Path (Med Microbiol), DTM\&H; A Brink, ${ }^{4}$ MB ChB, MMed (Clin Microbiol); M Blockman, ${ }^{5}$ BPharm, MB ChB, PG Dip Int Res Ethics, MMed (Clin Pharmacol)

${ }^{1}$ Department of Jurisprudence, School of Law, University of South Africa

${ }^{2}$ Department of Paediatrics, Faculty of Health Sciences, University of Cape Town, South Africa; and OpenBiome, Cambridge, Mass., USA

${ }^{3}$ Clinical Microbiology Department, Ampath Laboratories, Centurion, South Africa

${ }^{4}$ Division of Medical Microbiology, Department of Pathology, Faculty of Health Sciences, University of Cape Town, South Africa; and National Health Laboratory Service, Cape Town, South Africa

${ }^{5}$ Division of Clinical Pharmacology, Department of Medicine, Faculty of Health Sciences, University of Cape Town, South Africa

Corresponding author: M Labuschaigne (slabbmn@unisa.ac.za)

The legal regulation of faecal microbiota transplantation (FMT) in South Africa (SA) is currently unclear. The purpose of this article, the first of three in a series, is to explore the nature, role and clinical application of FMT in SA in order to determine, from a legal perspective, the appropriate regulatory pathways governing FMT as a procedure that may combine approaches for the treatment of drugs, human tissue for transplantation, or clinical treatment as part of the practice of medicine. FMT has been shown to be a novel, safe and effective treatment for recurrent Clostridioides difficile infection (CDI). Stool banks are instrumental in enabling access to FMT for patients and clinicians and help to catalyse research in the microbiome. However, the regulatory landscape in SA remains unclear. Microbial therapies such as FMT are necessary, especially in a time of rising microbiome-associated inflammatory diseases and increasing resistance to traditional antibiotics. FMT is now considered as part of the standard of care for recurrent CDI overseas, but is currently only being used for research purposes in a minority of clinical cases of CDI in SA. This article, which lays the foundation for consideration of this question in three parts, suggests that the relevant regulatory system would depend on the categorisation of human stool as tissue, the exact composition of the FMT, how it is administered to patients, and the relevant levels of manipulation of the stool for FMT-derived products.

S Afr Med J 2020;110(8):812-815. https://doi.org/10.7196/SAMJ.2020.v110i8.14563

The human body hosts a series of dynamic and diverse microbial communities consisting of bacteria, archaea, viruses and eukaryotes, collectively known as the human microbiome. The microbiome plays a critical role in health and disease and has been shown to influence various medical conditions, ranging from diabetes to multiple sclerosis, autism, cancer and inflammatory bowel disease. ${ }^{[1]}$

Globally there has been a rapid increase in research and scientific publications predominantly on the gastrointestinal (GIT) microbiome and its role in health, immunology and nutrition. Therapeutic applications are of great interest, and a successful example is faecal microbiota transplantation (FMT). This process involves the introduction of gut bacteria from healthy donors into patients, with the aim of repairing the abnormal microbial community in the recipient. The efficacy of FMT for treatment of recurrent Clostridioides difficile infection (CDI) is a crucial proof of the concept that therapeutic modification of the GIT microbiome can be an effective treatment modality for human disease. ${ }^{[2]}$ Other indications for FMT are also currently being explored. ${ }^{[3]}$

The legal regulation of microbiome-based therapy is difficult to categorise, however, as it poses an uncomfortable fit in terms of the South African (SA) legal framework governing the use of human tissue, as well as the legal regulation of medicines and medical devices. $^{[2]}$ More specifically, human stool banking or creation of a repository is critical to an FMT service. The purpose of this article is to explore the use of FMT as a microbiome-based therapy in SA by exploring the nature of FMT, followed by the role of FMT in clinical practice and then indicating the legal vacuum in which FMT currently exists.

The need for legal clarity on the regulation of FMT is underscored by a recent safety alert issued by the US Food and Drug Administration (FDA) regarding the transmission of a multidrug-resistant organism that resulted in two immunocompromised patients developing bacteraemia following an FMT. ${ }^{[4]}$ This incident led to the death of one patient, and subsequent investigation found that the donor had not been screened for extended-spectrum beta-lactamase organisms. ${ }^{[5-7]}$

\section{Understanding the role of the microbiome}

Microbial cells outnumber human cells by approximately three to one. ${ }^{[8]}$ The combined microbial genome encodes 500 times more genes than the human genome. The human microbiota refers to all the micro-organisms residing in the human body. The microbiome includes all the microbiota, and their genetic products - proteins, metabolites and RNA. ${ }^{[9,10]}$

The human gut has the largest collection of microbes, producing signals that influence host metabolic, immune and physiological functions. The five most abundant bacterial phyla found in healthy individuals are Bacteroidetes, Firmicutes, Actinobacteria, Proteobacteria and Verrucomicrobia. Within each phylum there are hundreds of bacterial species, each found in different abundances 
in healthy individuals. ${ }^{[1,12]}$ Gut bacterial lineages are highly variable, making a healthy core microbiome difficult to describe at organism phylum level. Shared microbial genes in the gut of different individuals indicate a common functional core microbiome at the gene level, ${ }^{[13]}$ implying that different microbes have similar metabolites or functions and the absence of a single bacterial species may not be impactful. ${ }^{[14]}$

A healthy microbiome consists of a diverse assembly of microbial species in specific anatomical niches carrying out their specific biomolecular function. High microbial alpha diversity is associated with host health and microbial stability to environmental pressures. Alpha diversity can be described as the number of different microbial taxa and their abundance in an ecosystem. ${ }^{[15]}$ Dysbiosis is a term that refers to disruption of the normal gut microbiome (e.g. a decrease in microbial alpha diversity) and is associated with various diseases such as obesity, ${ }^{[16]}$ inflammatory bowel disease, autoimmune disease, allergy and diabetes. ${ }^{[17]}$ Broad-spectrum antibiotics can decrease microbial diversity with variable recovery dynamics. When healthy gut microbes are diminished, they are unable to compete against pathogens wanting to colonise the gut. ${ }^{[18]}$ In addition, certain beneficial microbes that produce beneficial metabolites (e.g. short-chain fatty acids (SCFAs)) perform important metabolic functions (e.g. metabolism of bile salts), produce anti-inflammatory metabolites or enhance gut epithelial function, and may be lost due to antibiotics or disease.

There are several factors that influence the microbiome during early life. Significant ones include mode of delivery (normal vaginal delivery v. caesarean section), gestational age at delivery, ${ }^{[19]}$ infant feeding patterns (breastfed v. formula fed) and weaning foods, ${ }^{[20]}$ antibiotic use, ${ }^{[21]}$ and maternal factors such as health and diet. ${ }^{[13]}$ A recently published study that included the largest dataset of children born by either caesarean section or vaginal delivery demonstrated persistent significant differences in the microbial community composition associated with mode of delivery. ${ }^{[22]}$ The gut microbiome undergoes substantial changes or maturation during the first 3 years of life and contributes to the development and maturation of the child's immune system. By the age of 3 , the child's microbiome resembles that of an adult. ${ }^{[23]}$

The ongoing composition of the microbiome is influenced by host genetics to a limited extent and more significantly by environmental factors. Age, sex, geographical location, chronic disease, diet, medication (specifically antibiotics) and other environmental exposures (chemical or microbial) all play a role. Diet greatly shapes gut microbiota and may explain the geographical variation observed between populations on different continents. ${ }^{[18]}$

Gut-derived signalling molecules influence peripheral organs and metabolism. Signalling molecules can be part of the bacterial structure, such as lipopolysaccharide, which interacts with host receptors to produce an immunological response. Other important signalling molecules are byproducts of bacterial metabolism and are influenced by diet, such as SCFAs. Gut microbiota can be considered as a separate organ that influences the immune system, brain, lung, liver, skin, adipose tissue and metabolism. The microbiome-gut-brain axis refers to the myriad effects that the gut microbiota has on the brain and its functions. ${ }^{[10]}$

FMT is one modality that is used to manipulate the microbiome. Other techniques include probiotics, diet, phage therapy and antibiotics. Extensive data suggest that FMT can restore the healthy intestinal microbiome in a patient by enabling engraftment of donor microbes into recipients. ${ }^{[24]}$

C. difficile is an anaerobic, spore-forming, Gram-positive intestinal pathogen that is present in our environment and can asymptomatically colonise a healthy host. CDI occurs when bacterial toxins cause diarrhoea, and in certain patients this progresses to a life-threatening colitis. Dysbiosis of the gut microbiome caused by antibiotic use is the main risk factor for CDI. The first-line treatment for CDI is administration of antibiotics directed against CDI, but despite various dosing regimens for oral vancomycin, $20 \%$ of patients go on to develop recurrent CDI. Recent US and European guidelines now support the use of FMT to treat recurrent CDI. ${ }^{[24,25]}$ Meta-analysis of randomised controlled trials and case series indicates a cure rate of 80 - 90\% with a single treatment, but some patients will require more than one FMT. ${ }^{[26]}$ Current evidence suggests that FMT is a safe and well-tolerated procedure in patients with recurrent CDI. ${ }^{[26,27]}$

To date, a single USA-based stool bank, OpenBiome, has treated $>50000$ patients with recurrent CDI and currently supports $>30$ enrolling FMT clinical trials globally. This stool banking model (based on the blood banking model) is critical to an FMT service from both doctor/patient access and research perspectives. Prior to the expansion of stool banks, patients were required to identify their own donors who would need to be consented and screened by the treating doctor, a process that was prohibitively expensive and introduced ethical concerns around coercion and disclosure from patient-selected donors. ${ }^{[3,28]}$ In addition, the collection and processing of the material was unpleasant and beyond the scope of most doctors. These factors were reported as major barriers to access to FMT for both patients and doctors. ${ }^{[29]}$ The stool bank model removed many of these barriers and enabled almost universal access to FMT in the USA. ${ }^{[3]}$ In the stool bank model, prospective donors undergo a detailed clinical assessment aimed at ruling out those who are an infectious risk or have a personal or immediate family history of microbiome-mediated diseases such as autoimmune disease, allergy, neuropsychiatric disease, malignancy, etc. Thereafter, prospective donors undergo extensive blood and stool tests for potential pathogens. ${ }^{[30]}$

Those who pass this process are onboarded as stool donors and undergo repeat clinical and laboratory screening at 2-monthly intervals, in addition to brief clinical assessment at each donation, to ensure that there has been no acute change in the donor's health status. Stool is frozen in a cryopreservative buffer and stored at $-80^{\circ} \mathrm{C}$ until needed. In addition, a safety aliquot is retained from each sample that is donated. This safety aliquot can be used for pathogen testing during investigation of an adverse event. Owing to the theoretical risk of transfer of inflammatory disease from donors to recipients, stool banks aim to retain data and samples from donors for extended periods. ${ }^{[25]}$ The American Gastroenterological Association has established an FMT registry in the USA that aims to prospectively follow up patients, adult and paediatric, up to 10 years after FMT, with the aim of establishing long-term safety data.

\section{Regulatory challenges to date}

Regulatory challenges in the USA and Europe are one of the main reasons why FMT is not widely adopted among doctors. In 2013, the FDA announced that FMT would be regulated as a biological and as an investigational drug. ${ }^{[31]}$ An initial regulation was that an investigational new drug (IND) application was required for access for all disease indications. Following the landmark paper by Van Nood et al. ${ }^{[32]}$ in 2013, demonstrating significantly superior efficacy of FMT over placebo or standard of care in recurrent CDI, and subsequent pressure from providers and patients, the FDA chose to exercise enforcement discretion regarding the requirement for an IND requirement to treat recurrent CDI, thereby allowing clinicians and patients to access FMT. The use of FMT to treat any other disease indication would, however, require an IND application. 
Another significant barrier for doctors and patients was access to material for treatment. The study by Lee et al. , $^{[33]}$ which demonstrated similar efficacy of frozen v. fresh FMT, catalysed the development of the universal donor stool bank. In this model, donors could be thoroughly screened and material banked for convenient future use by doctors. ${ }^{[33]}$

In 2016, a draft guidance statement from the FDA raised a safety concern related to the use of banked stool samples. ${ }^{[31]}$ It proposed that stool banks would be required to submit an IND application to obtain and distribute stool. The draft guidance would not affect clinicians who were collecting and screening patient-selected donor stools. A safety concern of the FDA was that centralised stool banking may lead to a relatively small number of donors providing material to multiple patients. Potential transmission of infectious agents and other unidentifiable risks related to the microbiome of the few donors would reach many recipients. ${ }^{[31]}$

Criticism of the proposed regulation change was raised among both clinicians and consumers, and the draft guidance was never implemented. Owing to the ease of access to safe material using a stool bank, most medical facilities in the USA choose to use stool bank material for FMT. Stools for banking undergo extensive investigation for transmissible pathogens, as opposed to direct donation, where quality control is not possible. ${ }^{[31]}$ Stool banks offer a centralised point of reference for safety monitoring and regulatory oversight and reduce costs through economies of scale.

A recent framework has been proposed in the USA that allows the degree of manipulation of the donor faecal material to determine the regulatory category. ${ }^{[34,35]}$ Faecal material from a donor that is not manipulated or minimally manipulated (e.g. addition of a cryopreservative buffer or saline homogenisation) would be considered practice of medicine and not subject to federal regulation. When stools are manipulated and consist of defined bacterial populations, faecal material would fall under a traditional drug category. Stool banks would fall under a regulatory category like that of a tissue or blood bank.

Based on the successful US model of a stool bank of having treated $>50000$ patients with only two adverse events, the ideal scenario would be to replicate this model in the SA and African context, while at the same time adapting it to the local context and disease burden. In order to ensure that FMT is performed safely, and is accessible to doctors, patients and researchers, SA must carefully consider how best to regulate stool banks and FMT.

\section{Nature of FMT for the purpose of legal regulation}

It seems as if stool donations would fall within the scope of the definition of 'biological material' as defined in the 2012 Regulations on the Use of Human Biological Material, ${ }^{[36]}$ promulgated in terms of the National Health Act 61 of 2003 (NHA), which refers to biological material as 'material from a human being. The use of stool samples in FMT is not covered directly by these regulations or any other provisions in the NHA itself. The nature of FMT is equally unclear, as it is difficult to determine whether the procedure should be regarded in law as a medicine or a medical treatment.

The nature of FMT is unique and different from conventional medicines or therapies. It may include characteristics and components of a 'health service' in terms of the NHA, which include medical treatment, or a 'medicine' as described in the Medicines and Related Substances Control Act 101 of 1965. Each of these has different legal and ethical considerations, depending on the nature of the procedure.

If administered orally by way of swallowing capsules, FMT will arguably be regarded as a medicine. The administration of FMT via a nasoduodenal tube or colonoscopy resembles medical treatment. The confusion regarding the exact nature of FMT is also evident from the FDA's position on the issue, which has shifted a few times in recent years. As mentioned above, the FDA currently classifies FMT as a drug (e.g. a live biotherapeutic product), subject to enforcement discretion of not requiring an IND for doctors performing the procedure for patients with recurrent CDI unresponsive to standard therapies. ${ }^{[31]}$

Regulatory systems may decide to regulate FMT as both a product and a procedure that combines approaches for the treatment of drugs, blood and human tissue for transplantation, or view it as part of the practice of medicine (e.g. clinical treatment). The regulatory pathway would depend on the exact composition of the FMT and the relevant formulations or levels of manipulation of the stool, which may range from basic frozen stool to capsules containing frozen or lyophilised stool, or defined consortia of bacteria. ${ }^{[35]}$ Stool-based products containing specific bacterial strains aimed at treating specific diseases involve a higher level of manipulation and therefore a more targetspecific approach.

In the most elementary FMT procedure, faeces are liquefied, and saline or glycerol (e.g. a cryopreservative) added, which points to minimal manipulation. The FDA's definition of 'minimal manipulation' of cells or non-structural tissue in the case of the transplantation of human cells and tissues refers to 'processing that does not alter the relevant biologic characteristics of cells or tissue. ${ }^{[37]}$ This prompts the question regarding what the original relevant characteristics of stool are, and how one knows that these have changed. Ossorio and Zhou ${ }^{[38]}$ rightly remark that if the scientific community has not adequately characterised the health-related functions of microbes in complex communities, considering that 'people's microbial communities are highly dynamic, then clinicians and regulators lack metrics for determining when a microbial community's relevant properties have been altered by manipulation?

According to Hoffmann et al. ${ }^{[35]}$ the degree of manipulation of stool may vary as follows from the least to the most manipulated: (i) fresh stool transferred from an individual donor; (ii) frozen filtered stool from a stool bank; (iii) concentrated stool microbiota in capsule form; (iv) biologically sourced, purified microbial groups of specific bacterial strains; and $(v)$ cultured bacterial mixture delivered in oral pill form.

In the USA, stool that is extensively manipulated, isolated or combined with other components, or used for novel functions, would therefore be regulated under section 351 of the Public Health Services Act (2012) and the Federal Food, Drug, and Cosmetic Act as biological drugs or devices requiring premarket approval under Title 21 of the Code of Federal Regulations (1271.20), as stated above. Determining whether manipulated stool is a product or a procedure may also require characterisation of the stool (e.g. to document the structure and properties of the material). The regulatory process for live biotherapeutic products traditionally requires characterisation of all the microbes in the faecal material, which would require wholecommunity genome sequencing of all the microbes contained in the stool. ${ }^{[28]}$ This would not only be scientifically challenging but impossible, because human stool differs biologically and chemically considerably from one person to the next, rendering the regulatory requirement of consistency in product composition unfeasible. ${ }^{[28]}$

There is no doubt that FMT using single-donor stool (e.g. from a friend or relative) creates fewer risks, and it is for this reason that the recent draft FDA guidance document makes provisions for an 'enforcement discretion' when 'the stool donor and stool are qualified by screening and testing performed under the direction of the licensed healthcare provider for the purpose of providing the FMT product for treatment of the patient. ${ }^{[37]}$ The same cannot be said for 
public health risks associated with universal donors or pooled stool. In the case of pooled stool from multiple donors, it is possible that unknown interactions between different microbial communities and increased risk of infection may arise. ${ }^{[28]}$ The complexity with pooled stool is that it is difficult to trace the source of infection back to the actual donor. Ossorio and Zhou, ${ }^{[38]}$ however, in response to Hoffmann et al. ${ }^{[35]}$ maintain that if stool composition is dynamic and difficult to characterize, then a minimally-manipulated stool product for allogeneic transplant is possibly the most dangerous type of microbial product for a recipient. Compared to highly-manipulated but better characterized stool-derived products, stool from an asymptomatic donor is more likely to contain novel or uncharacterized viruses, bacteria, or protozoa that could be pathogenic, particularly for an already sick recipient.' However, the role of minimally manipulated stool (transplantation of the entire microbiome community from a healthy donor) should not be dismissed, as there may be currently uncharacterised features of stool (e.g. viral, fungal components or metabolites) that could convey beneficial effects in some diseases. In addition, the use of whole-stool FMT will provide scientific data to inform the future selection of specific beneficial microbes necessary to develop defined consortia.

It is clear from this discussion that the uncertainties regarding the nature of stool and stool-derived products will continue to hamper their effective classification.

\section{Conclusions}

FMT has been shown to be a novel, safe and effective treatment for recurrent CDI. Stool banks are instrumental in enabling access to FMT for patients and clinicians and help to catalyse research in the microbiome. However, the regulatory landscape in SA remains unclear. Microbial therapies such as FMT are necessary, especially in a time of rising microbiome-associated inflammatory diseases and increasing resistance to traditional antibiotics. FMT is now considered as part of standard of care for recurrent CDI overseas, but is currently only being used for research purposes or in a minority of clinical cases of CDI in SA. The advantages of the procedure are not disputed, but what is needed is clarity on the regulatory framework in which FMT is performed. This article recommends that the relevant regulatory pathway for FMT would depend on the exact composition of the FMT and the relevant formulations or levels of manipulation of the stool. In part 2 of this series of articles, the legal categorisation of human stool as human tissue will be considered, as well as the application thereof in clinical practice. This will involve an analysis of the relevant legal framework to determine how FMT will be regulated in SA in future. The fact that FMT is still in a research phase in SA also has peculiar legal consequences that need to be explored. If the stool donations are processed and transformed into products, such as capsules, the legal framework may change completely. These issues will be interrogated further in subsequent parts of this series of articles.

Declaration. None.

Acknowledgements. None.

Author contributions. Equal co-authors.

Funding. None.

Conflicts of interest. SB is employed by Finch Therapeutics, Somerville, Mass., USA, a company developing microbial therapies related to the treatments described in these articles.

1. Wang H, Wei C-X, Min L, Ling-Yun Zhu L-Y. Good or bad: Gut bacteria in human health and diseases. Biotechnol Biotechnol Equip 2018;32(5):1075-1080. https://doi.org/10.1080/13102818.20 18.1481350
2. Fredericks E, Hoosein E, Brink A. The case for stool banks in South Africa. S Afr Med J 2019;109(8):546547. https://doi.org/10.7196/SAMJ.2019.v109i8.14169

3. Panchal P, Budree S, Scheeler A, et al. Scaling safe access to fecal microbiota transplantation: Past, present, and future. Curr Gastroenterol Rep 2018;20(4):14. https://doi.org/10.1007/s1 1894-018-0619-8

4. US Food and Drug Administration. FDA in brief: FDA warns about potential risk of serious infections caused by multi-drug resistant organisms related to the investigational use of fecal microbiota for caused by multi-drug resistant organisms related to the investigational use of fecal microbiota for
transplantation. https://www.fda.gov/news-events/fda-brief/fda-brief-fda-warns-about-potential-risktransplantation. https://www.fda.gov/news-events/fda-brief/fda-brief-fda-warns-about-p
serious-infections-caused-multi-drug-resistant-organisms (accessed 30 September 2019).

serious-infections-caused-multi-drug-resistant-organisms (accessed 30 September 2019).
5. Kuijper EJ, Allegretii J, Hawkey P, et al. A necessary discussion of transmission of multidrug-resistant organisms through faecal microbiota transplantations. Lancet Infect Dis 2019;19(11):1161-1162. https:// doi.org/10.1016/S1473-3099(19)30545-6

6. DeFilipp Z, Bloom PP, Soto MT, et al. Drug-resistant $E$. coli bacteremia transmitted by fecal microbiota transplant. N Engl J Med 2019;381:2043-2050. https://doi.org/10.1056/NEJMoa1910437

Blaser MJ. Fecal microbiota transplantation for dysbiosis - predictable risk. N Engl J Med 2019:381(21):2064-2066. https://doi.org/10.1056/NEJMe1913807

8. Rosner J. Ten times more microbial cells than body cells in humans? Microbe 2014;9(2):47-47. https:// doi.org/10.1128/microbe. 9.47 .2

9. Turnbaugh PJ, Ley RE, Hamady M, Fraser-Liggett CM, Knight R, Gordon JI. The human microbiome project. Nature 2007;449(7164):804-810. https://doi.org/10.1038/nature06244

10. Schroeder BO, Backhed F. Signals from the gut microbiota to distant organs in physiology and disease. Nat Med 2016;22(10):1079-1089. https://doi.org/10.1038/nm.4185

11. Human Microbiome Jumpstart Reference Strains Consortium: Nelson KE, Weinstock GM, Highlander SK, et al. A catalog of reference genomes from the human microbiome. Science 2010;328(5981):994-999. https://doi.org/10.1126/science.1183605

12. Costea PI, Hildebrand F, Arumugam M, et al. Enterotypes in the landscape of gut microbial community composition. Nat Microbiol 2018;3(1):8-16. https://doi.org/10.1038/s41564-017-0072-8

13. Turnbaugh PJ, Gordon JI. The core gut microbiome, energy balance and obesity. J Physiol 2009:587(Pt 17):4153-4158. https://doi.org/10.1113/jphysiol.2009.174136

14. Human Microbiome Project Consortium. Structure, function and diversity of the healthy human microbiome. Nature 2012;486(7402):207-214. https://doi.org/10.1038/naturel1234
miturel

15. Knight R, Callewaert C, Marotz C, et al. The microbiome and human biology. Annu Rev Genomics Hum . Knight R, Callewaert C, Marotz C, et al. The microbiome and human biology. Ann
Genet 2017;18(1):65-86. https://doi.org/10.1146/annurev-genom-083115-022438

16. Turnbaugh PJ, Hamady M, Yatsunenko T, et al. A core gut microbiome in obese and lean twins. Nature 2009:457(7228):480-484, Yatsunenko T, et al. A core gut mis

17. Editorial. After the Integrative Human Microbiome Project, what's next for the microbiome community? . Editorial. After the Integrative Human Microbiome Project, what's next for
Nature 2019;569(7758):599. https://doi.org/10.1038/d41586-019-01674-w

18. Lloyd-Price J, Abu-Ali G, Huttenhower C. The healthy human microbiome. Genome Med 2016;8(1):51. https://doi.org/10.1186/s13073-016-0307-y

19. Gibson MK, Wang B, Ahmadi S, et al. Developmental dynamics of the preterm infant gut microbiota and antibiotic resistome. Nat Microbiol 2016;1:16024. https://doi.org/10.1038/nmicrobiol.2016.24

20. Walker RW, Clemente JC, Peter I, Loos RJF. The prenatal gut microbiome: Are we colonized with bacteria in utero? Pediatr Obes 2017;12(Suppl 1):3-17. https://doi.org/10.1111/ijpo.12217

21. Zhang M, Differding MK, Benjamin-Neelon SE, Ostbye T, Hoyo C, Mueller NT. Association of prenatal antibiotics with measures of infant adiposity and the gut microbiome. Ann Clin Microbiol Antimicrob 2019;18(1):18. https://doi.org/10.1186/s12941-019-0318-9

22. Shao Y, Forster SC, Tsaliki E, et al. Stunted microbiota and opportunistic pathogen colonization in caesarean-section birth. Nature 2019;574:117-121. https://doi.org/10.1038/s41586-019-1560-1

23. Yatsunenko T, Rey F, Manary M, et al. Human gut microbiome viewed across age and geography. Nature 2012;486:222-228. https://doi.org/10.1038/nature11053

24. McDonald LC, Gerding DN, Johnson S, et al. Clinical Practice Guidelines for Clostridium difficile Infection in Adults and Children: 2017 update by the Infectious Diseases Society of America (IDSA) and Society for Healthcare Epidemiology of America (SHEA). Clin Infect Dis 2018;66(7):987-994. https:// doi.org/10.1093/cid/cix1085

25. Cammarota G, Ianiro G, Tilg H, et al. European consensus conference on faecal microbiota transplantation in clinical practice. Gut 2017;66(4):569-580. https://doi.org/10.1136/gutjnl-2016-313017

26. Moayyedi P, Yuan Y, Baharith H, Ford AC. Faecal microbiota transplantation for Clostridium difficileassociated diarrhoea: A systematic review of randomised controlled trials. Med I Aust 2017:207(4):166172. https://doi.org/10.5694/mja17.00295

27. Quraishi MN, Widlak M, Bhala N, et al. Systematic review with meta-analysis: The efficacy of faecal microbiota transplantation for the treatment of recurrent and refractory Clostridium difficile infection. Aliment Pharmacol Ther 2017;46(5):479-493. http://doi.wiley.com/10.1111/apt.14201

28. Allegretti JR, Kassam Z, Osman M, Budree S, Fischer M, Kelly CR. The 5D framework: A clinical primer for fecal microbiota transplantation to treat Clostridium difficile infection. Gastrointest Endosc
peral primer for fecal microbiota transplantation to treat Clostri
2017;87(1):18-29. https://doi.org/10.1016/j.gie.2017.05.036

29. Bakken JS, Polgreen PM, Beekmann SE, Riedo FX, Streit JA. Treatment approaches including fecal Bakken JS, Polgreen PM, Beekmann SE, Riedo FX, Streit JA. Treatment approaches including fecal
microbiota transplantation for recurrent Clostridium difficile infection (RCDI) among infectious disease microbiota transplantation for recurrent Clostridium difficile infection (RCD) among
physicians. Anaerobe 2013;24:20-24. https://doi.org/10.1016/j.anaerobe.2013.08.007

30. Kassam Z, Dubois N, Ramakrishna B, et al. Donor screening for fecal microbiota transplantation. N Engl Kassam Z, Dubois N, Ramakrishna B, et al. Donor screening for fecal
J Med 2019;381:2070-2072. https://doi.org/10.1056/NEJMc1913670

31. US Food and Drug Administration. Enforcement policy regarding investigational new drug requirements for use of fecal microbiota for transplantation to treat Clostridium difficile infection not responsive to standard therapies: Draft guidance for industry. March 2016. https://www.fda.gov/regulatoryinformation/search-fda-guidance-documents/enforcement-policy-regarding-investigational-new-drugrequirements-use-fecal-microbiota-0 (accessed 24 June 2020)

32. Van Nood E, Vrieze A, Nieuwdorp M, et al. Duodenal infusion of donor feces for recurrent Clostridium difficile. N Engl J Med 2013;368(5):407-415. https://doi.org/10.1056/NEJMoa1205037

33. Lee CH, Steiner T, Petrof EO, et al. Frozen vs fresh microbiota transplantation and clinical resolution of diarrhea in patients with recurrent Clostridium difficile infection: A randomized clinical trial. JAMA 2016;315(2):142-149. https://doi.org/10.1001/jama.2015.18098

34. Hoffmann D, Palumbo F, Ravel J, Roghmann MC, Rowthorn V, von Rosenvinge E. Improving regulation of microbiota transplants. Science 2017;358(6369):1390-1391. https://doi.org/10.1126/science.aaq0034

35. Hoffmann DE, Palumbo FB, Ravel J, Rowthorn V, von Rosenvinge E. A proposed definition of microbiota transplantation for regulatory purposes. Gut Microbes 2017;8(3):208-213. https://doi.org/10.1080/1949 0976.2017.1293223

36. National Department of Health, South Africa. National Health Act 61 of 2003. Regulations relating to the use of human biological material. Government Gazette 35099, 2 March 2012. (Published under Government Notice R177.) https://www.gov.za/sites/default/files/gcis_document/201409/35099rg9699 gon177.pdf (accessed 6 July 2020).

37. US Food and Drug Administration. Regulatory considerations for human cells, tissues, and cellular and tissue-based products: Minimal manipulation and homologous use guidance for industry and Food and Drug Administration Staff. December 2017. https://www.fda.gov/media/124138/download (accessed 24 June 2020).

8. Ossorio PN, Zhou Y. Regulating stool for microbiota transplantation. Gut Microbes 2019;10(2):105108. https://doi.org/10.1080/19490976.2018.1502537. 